\title{
EXISTENCE OF QUASICONFORMAL MAPPINGS BETWEEN RIEMANNIAN MANIFOLDS
}

\author{
By Mitsuru Nakai and Hiroshi Tanaka
}

\section{Introduction.}

In 1960 the first named author [8] proved that two Riemann surfaces are quasiconformally equivalent if and only if their Royden algebras are isomorphic. This result was extended to higher dimensions: to higher dimensional Euclidean domains by L. G. Lewis [6] and to Riemannian manifolds by J. Lelong-Ferrand [5]. These results show that if two Riemannian manifolds $M$ and $N$ are quasiconformally equivalent, then their Royden compactifications $M^{*}$ and $N^{*}$ are homeomorphic. The question aries whether the converse is true, that is, whether a homeomorphism from $M^{*}$ to $N^{*}$ can always be raised to a quasiconformal mapping from $M$ to $N$.

In this paper we shall prove that the question is true in a neighborhood of ideal boundary of $M$, that is, if there is a homeomorphism $f$ of $M^{*}$ onto $N^{*}$, then there exists a compact subset $K$ of $M$ such that the restriction of $f$ to each component of $M-K$ is quasiconformal. Furthermore, for Riemann surfaces, we can find a quasiconformal mapping from $M$ to $N$. However we do not know whether this is valid for higher dimensional cases.

\section{Notation and terminology}

We denote by $R^{n}$ the $n$-dimensional Euclidean space whose points $x$ are $n$-tuple $x=\left(x_{1}, x_{2}, \cdots, x_{n}\right)$ of real numbers $(n \geqq 1)$. The distance between $x=$ $\left(x_{1}, \cdots, x_{n}\right)$ and $y=\left(y_{1}, \cdots, y_{n}\right)$ is denoted by

$$
|x-y|=\left(\sum_{\imath=1}^{n}\left|x_{i}-y_{i}\right|^{2}\right)^{1 / 2} \text {. }
$$

We denote by $\omega_{n-1}$ the $(n-1)$-dimensional Lebesgue measure of the unit sphere $\left\{x \in R^{n} ;|x|=1\right\}$.

\section{Riemannian manifolds}

Let $M$ be a connected separable, orientable $n$-idmensional ( $n \geqq 2)$ differentiable manifold of class $C^{1}$ with fundamental metric tensor

Received October 15, 1980. 


$$
G=\left(\begin{array}{c}
g_{11} \cdots \cdot g_{1 n} \\
\cdots \cdots \cdot g_{n n} \\
g_{{ }_{n}} \cdots \cdot g_{n n}
\end{array}\right)
$$

satisfying the following conditions:

In each parametric ball or cube $B=(B, \phi)$ with local parameter $\phi(p)=$ $\left(x^{1}, \cdots, x^{n}\right)(p \in B)$, the local expressions $g_{\imath \jmath}(x)$ of $g_{\imath \jmath}(\imath, \jmath=1, \cdots, n)$ are continuous functions of $x=\left(x^{1}, \cdots, x^{n}\right)$ in $\phi(B)$ and there exists a finite constant $k_{B} \geqq 1$ such that

$$
k_{B}{ }^{-1} \cdot \sum_{\imath=1}^{n}\left(\xi^{\imath}\right)^{2} \leqq \sum_{\imath, j=1}^{n} g_{\imath \jmath}(x) \xi^{\imath} \xi^{\jmath} \leqq k_{B} \cdot \sum_{\imath=1}^{n}\left(\xi^{i}\right)^{2}
$$

for every $x$ in $\phi(B)$ and for every $n$-tuple $\left(\xi^{1}, \cdots, \xi^{n}\right)$ of real numbers. We can, therefore, consider the inverse matrix $G^{-1}$ of $G$. We set

$$
G^{-1}=\left(\begin{array}{c}
g^{11} \cdots \cdot g^{1 n} \\
\cdots \cdots \cdot g^{n n}
\end{array}\right), \quad g=\operatorname{det} G .
$$

Then it is known that

$$
k_{B}{ }^{-1} \cdot \sum_{i=1}^{n}\left(\eta_{\imath}\right)^{2} \leqq \sum_{\imath, j=1}^{n} g^{\imath j}(x) \eta_{\imath} \eta_{j} \leqq k_{B} \cdot \sum_{i=1}^{n}\left(\eta_{\imath}\right)^{2}
$$

for every $n$-tuple $\left(\eta_{1}, \cdots, \eta_{n}\right)$ of real numbers and that

$$
k_{B}{ }^{-n} \leqq g \leqq k_{B}{ }^{n} \text {. }
$$

In terms of local parameter $x=\left(x^{1}, \cdots, x^{n}\right)$, the line element $d s$ on $M$ is given by $d s^{2}=\sum_{\imath, j=1}^{n} g_{\imath j}(x) d x^{\imath} d x^{\jmath}$, and since $g_{\imath j}(x)$ are continuous, the line integral $\int_{\gamma} d s$ along a rectifiable curve $\gamma$ in $M$ can be defined. Therefore the natural distance $d_{M}(p, q)$ of two points $p$ and $q$ in $M$ is given by

$$
d_{M}(p, q)=\inf \int_{r} d s
$$

where the infinimum is taken with respect to all rectifiable curves $\gamma$ in $M$ joining $p$ and $q$.

We can find a covering $\{B\}$ of $M$ consisting of local parametric balls or cubes $B$ and a constant $\tau_{M} \in(1, \infty)$ such that

$$
1 \leqq k_{B} \leqq \tau_{M}
$$

for every $B$ of the covering. Thus we fix such a covering $\{B\}$ of a manifold $M$ and a constant $\tau_{M}$ once for all.

By the aid of (1) we have the following lemma.

LEMMA 1 (cf. [5]). If $B=(B, \phi)$ is a parametric ball on $M$, then

$$
\left(k_{B}\right)^{-1 / 2}|\phi(q)-\phi(p)| \leqq d_{M}(p, q) \leqq\left(k_{B}\right)^{1 / 2}|\phi(q)-\phi(p)|
$$


for all $p$ and $q$ in $B$.

In particular, if $\phi(p)=0$, then, for sufficiently small $r, t>0$, we have

(i) $d_{M}(p, q)=r$ implies $k_{B}{ }^{-1 / 2} r \leqq|\phi(q)| \leqq k_{B}{ }^{1 / 2} r$,

(ii) $|x|=$ t $\imath m p l i e s ~ k_{B}{ }^{-1 / 2} t \leqq d_{M}\left(p, \phi^{-1}(x)\right) \leqq k_{B}{ }^{1 / 2} t$.

\section{ACL functions and Dirichlet integrals}

A continuous function $f$ defined on a cube $D: a^{\imath}<x^{\imath}<b^{\imath}(\imath=1, \cdots, n)$ in $R^{n}$ is said to be absolutely continuous on lines (abbreviated as $A C L$ ) if it is absolutely continuous on almost all line segments parallel to coordinate axes; explicitly, if we denote by $D_{\imath}$ the face of $D$ given by $x^{2}=a^{2}$, then the function $f\left(\xi+\eta e_{\imath}\right)$, $e_{\imath}=\left(\delta^{i 1}, \cdots, \delta^{2 n}\right)$, is absolutely continuous in $\eta \in\left(a^{\imath}, b^{i}\right)$ for almost all $\xi \in D_{\imath}$ with respect to the $(n-1)$-dimensional Lebesgue measure $(i=1, \cdots, n)$. Let $G$ be a domain in $R^{n}$ and $f$ be a funciton defined on $G$. Then $f$ is said to be $A C L$ if the restriction $f \mid D$ of $f$ to $D$ is $A C L$ for all cubes $D$ contained in $G$.

A function $f$ defined on a parametric ball $B=(B, \phi)$ on $M$ is said to be $A C L$ if $f \circ \phi^{-1}$ is $A C L$ in $\phi(B)$. Furthermore a function $f$ defined on $M$ is said to be $A C L$ if the restriction $f \mid B$ of $f$ to $B$ is $A C L$ for all parametric balls $B$ on $M$. For such a function $f$ on $M$ the Dirichlet integral $D_{M}(f)$ of $f$ is defined by

$$
D_{M}(f)=\int \cdots \int_{M}\left(\sum_{\imath, j=1}^{n} g^{\imath \jmath}(x) \frac{\partial f}{\partial x^{2}}(x) \frac{\partial f}{\partial x^{j}}(x)\right)^{n / 2} \sqrt{g(x)} d x^{1} \cdots d x^{n} .
$$

It may or may not be finite.

For an $A C L$ function $f$ defined on a domain $G$ in $R^{n}$ we define the Euclidean Dirichlet integral of $f$ by

$$
\|f\|_{G}=\int \cdots \int_{G}\left(\sum_{i=1}^{n}\left(\frac{\partial f}{\partial x^{2}}(x)\right)^{2}\right)^{n / 2} d x^{1} \cdots d x^{n} .
$$

Then we have the following lemma by the aid of (2) and (4).

LEMma 2. Let $f$ be an $A C L$ function defined on a parametric ball $B=(B, \phi)$ in $M$. Then we have

$$
k_{B}{ }^{-n}\left\|f \circ \phi^{-1}\right\|_{\dot{\varphi}^{\prime}(B)} \leqq D_{B}(f) \leqq k_{B}{ }^{n}\left\|f \circ \phi^{-1}\right\|_{\zeta(B)} .
$$

\section{Conformal capacity of a ring}

A non-empty open subset $A$ of a Riemannian manifold $M$ is called a generalized ring if the complement of $A$ consists of two non-empty closed subsets $C_{0}$ and $C_{1}$ of $M$ with $C_{0} \cap C_{1}=\emptyset$. In this case we write $A=R\left(C_{0}, C_{1} ; M\right)$. In particular if $A$ is relatively compact domain on $M$, both $C_{0}$ and $C_{1}$ are connected and $C_{1}$ is compact, then we say that $A$ is a ring. Then following $C$. Loewner [7] (cf. [2], [3]) we define the (conformal) capacity of a generalized ring. 
Definition. For a generalized ring $A=R\left(C_{0}, C_{1} ; M\right)$, we define

$$
C_{M}(A)=C_{M}\left[R\left(C_{0}, C_{1} ; M\right)\right]=\inf D_{M}(f),
$$

where $f$ run over all $A C L$ functions $f$ on $M$ such that $f=\imath$ on $C_{\imath}(i=0,1)$. If there is no such a funciton $f$, then we define $C_{M}(A)=\infty$. Furthermore, in the case $M=R^{n}$, we write $C_{M}(A)=C(A)$.

Let $A^{\imath}=R\left(C_{0}{ }^{(i)}, C_{1}{ }^{(i)} ; M\right)$ be two generalized rings $(\imath=1,2)$. If $C_{0}{ }^{(2}{ }^{2} \subset C_{0}{ }^{(1)}$ and $C_{1}{ }^{(2)} \subset C_{1}{ }^{(1)}$, then we write $A^{1} \leqq A^{2}$.

The following properties are immediate consequences of the definition of capacity and Lemma 2 except for (d).

Properties of a capacity:

(a) $C_{M}\left[R\left(C_{0}, C_{1} ; M\right)\right]=C_{M}\left[R\left(C_{1}, C_{0} ; M\right)\right]$.

(b) If $A \leqq A^{\prime}$, then $C_{M}(A) \geqq C_{M}\left(A^{\prime}\right)$.

(c) Let $A$ be a ring on $M$ which is contained in a parametric ball $B=$ $(B, \phi)$. Then

$$
k_{B}{ }^{-n} C(\phi(A)) \leqq C_{M}(A) \leqq k_{B}{ }^{n} C(\phi(A)) .
$$

(d) If $0<a<b<\infty$, then

$$
C(\{a<|x|<b\})=\frac{\omega_{n-1}}{(\log (b / a))^{n-1}} \quad(\text { cf. [12]). }
$$

Lemma 3. Let $p$ be a point in a parametric ball $B=(B, \phi)$. Let $a, b$ be real numbers such that $0<a<b<\infty$ and $A=\left\{q \in M ; a<d_{M}(p, q)<b\right\}$ is a ring in B. Then

$$
k_{B}{ }^{-n} \frac{\omega_{n-1}}{\left(\log k_{B}(b / a)\right)^{n-1}} \leqq C_{M}(A) \leqq k_{B}{ }^{n} \frac{\omega_{n-1}}{\left(\log k_{B}^{-1}(b / a)\right)^{n-1}} .
$$

The last inequality is valid if $b / a>k_{B}$.

Proof. We may assume that $\phi(p)=0$. If $b / a>k_{B}$, then it follows from (i) in Lemma 1 that

$$
\left\{k_{B}{ }^{-1 / 2} a<|x|<k_{B}{ }^{1 / 2} b\right\} \geqq \phi(A) \geqq\left\{k_{B}{ }^{1 / 2} a<|x|<k_{B}{ }^{-1 / 2} b\right\} .
$$

This completes the proof.

Let $\left\{A_{\jmath}=R\left(C_{0, \jmath}, C_{1, \jmath} ; M\right)\right\}_{\jmath=1}^{\infty}$ be a family of rings on $M$. We say that $\left\{A_{j}\right\}_{j=1}^{\infty}$ is a distinguished family of rings on $M$ if

$$
\left(A \jmath \cup C_{1, \jmath}\right) \cap\left(A_{k} \cup C_{1, k}\right)=0 \quad \text { if } \jmath \neq k .
$$

Then we have the following lemma.

LEMMA 4. Let $\left\{A_{\jmath}\right\}_{\jmath=1}^{\infty}$ be a distinguished family of rings on $M$. Let $C_{0}=$ $\bigcap_{\jmath=1}^{\infty} C_{0, \jmath}$ and $C_{1}=\bigcup_{\jmath=1}^{\infty} C_{1, \jmath}$. If $A=R\left(C_{0}, C_{1} ; M\right)$ is a generalized ring, then 


$$
C_{M}(A)=\sum_{\jmath=1}^{\infty} C_{M}\left(A_{\jmath}\right) .
$$

The following theorem is due to J. Väisälä.

Theorem 1 (cf. Theorem 11. 9 in [12]). Suppose that $A=R\left(C_{0}, C_{1} ; R^{n}\right)$ is a ring and that $c \in C_{0}$ and $a, b \in C_{1}$. Then

$$
C(A) \geqq \mathscr{H}_{n}\left(\frac{|c-a|}{|b-a|}\right),
$$

where $\mathcal{H}_{n}(r)$ is a positive constant depending only on $r>0$ and $n$.

\section{Homeomorphism} set

Definition. Let $f: M \rightarrow N$ be a homeomorphism. For $p \in M$ and $r>0$, we

$$
\begin{aligned}
& l(p, f, r)=\inf _{d_{M}(p, q)=r} d_{N}(f(p), f(q)), \\
& L(p, f, r)=\sup _{d_{M}(p, q)=r} d_{N}(f(p), f(q)), \\
& A^{*}(p, r)=\left\{q^{\prime} \in N ; l(p, f, r)<d_{N}\left(f(p), q^{\prime}\right)<L(p, f, r)\right\} .
\end{aligned}
$$

Proposition. If $f^{-1}\left(A^{*}(p, r)\right)$ is contained in $B=(B, \phi)$, then

$$
C_{M}\left[f^{-1}\left(A^{*}(p, r)\right)\right] \geqq k_{B}{ }^{-n} \mathscr{H}_{n}\left(k_{B}{ }^{2}\right)>0 .
$$

In partıcular, if $l(p, f, r)=L(p, f, r)$, then we set $C_{M}\left[f^{-1}\left(A^{*}(p, r)\right)\right]=\infty$.

Proof. We may assume that $l(p, f, r) \neq L(p, f, r)$. Then there exist $p_{1}, p_{2}$ $\in B$ such that $d_{N}\left(f(p), f\left(p_{1}\right)\right)=l(p, f, r)$ and $d_{N}\left(f(p), f\left(p_{2}\right)\right)=L(p, f, r)$. It follows from (c) that

$$
C_{M}\left[f^{-1}\left(A^{*}(p, r)\right)\right] \geqq k_{B}{ }^{-n} C\left[\phi\left(f^{-1}\left(A^{*}(f, r)\right)\right)\right]=(*) .
$$

If we set $\phi\left(p_{\imath}\right)=x_{\imath}(i=1,2)$, then it follows from (i) in Lemma 1 and Theorem 1 that

$$
\left({ }^{*}\right) \geqq k_{B}{ }^{-n} \mathscr{H}_{n}\left(\frac{\left|x_{2}\right|}{\left|x_{1}\right|}\right) \geqq k_{B}{ }^{-n} \mathscr{H}_{n}\left(k_{B}{ }^{2}\right)>0
$$

\section{Quasiconformal mappings on Riemannian manifolds}

Let $M$ and $N$ be connected separable, orientable $n$-dimensional $(n \geqq 2)$ differentiable manifolds of class $C^{1}$. The tangent bundle of $M$ is denoted by $T M$. The derivative of a differentiable mapping $f: M \rightarrow N$ is a fibre mapping $D f: T M$ $\rightarrow T N$ and the norm of $D f$ is denoted by $\|D f\|$. The Jacobian of $f$ at $p \in M$ is denoted by $J_{f}(P)=\operatorname{det} D f(p)$. 
We say that $f: M \rightarrow N$ is an $A C L^{n}$-mapping if, for any parametric balls $B=(B, \phi)$ on $M$ and $B^{\prime}=\left(B^{\prime}, \psi\right)$ on $N$ such that $f(B) \subset B^{\prime}, \psi \circ f \circ \phi^{-1}$ is an $A C L$ mapping and the partial derivatives of $\psi^{\circ} \circ \circ \phi^{-1}$ are locally $L^{n}$-integrable on $\phi(B)$. Then $f$ has a fibre mapping $D f$ almost everywhere on $M$.

Definition. A homeomorphism $f: M \rightarrow N$ is called a quasiconformal mapping if it is an $A C L^{n}$-mapping and if there exists a finite constant $K(\geqq 1)$ such that

$$
\|D f\|^{n} \leqq K \cdot\left|J_{f}\right|
$$

almost everywhere in $M$.

For a homeomorphism $f: M \rightarrow N$, we set

$$
H(p, f)=\varlimsup_{r \rightarrow 0} \frac{L(p, f, r)}{l(p, f, r)} \quad(p \in M) .
$$

Since the theory of quasiconformal mappings between Euclidean domains obviously carries over to Riemannian manifolds, we obtain the following theorem (cf. F. Gehring [3]).

THEOREM 2. Let $f: M \rightarrow N$ be an $A C L^{n}$-homeomorphism. Then $f$ is a quastconformal mapping if and only if $H(p, f)$ is bounded.

For a homeomorphism $f: M \rightarrow N$ we have the following theorem.

THEOREM 3. $f$ is a quasiconformal mapping if and only if there exists a finite constant $\alpha>0$ with the following property:

For every $p \in M$, there is $r(p)>0$ such that $\left\{q^{\prime} \in N ; d_{N}\left(f(p), q^{\prime}\right) \leqq r(p)\right\}$ is compact in $N$ and such that $C_{N}\left(A^{*}(p, r)\right) \geqq \alpha$ for all $r(0<r \leqq r(p))$.

Proof. Suppose there is a constant $K(1 \leqq K<\infty)$ such that $H(p, f) \leqq K$ for all $p \in M$. Then, for any $\varepsilon>0$ and $p \in M$, there exists $r(p)>0$ such that $F=$ $\left\{q \in M ; d_{M}(p, q) \leqq r(p)\right\}$ is compact in $M$ and

$$
1 \leqq \frac{L(p, f, r)}{l(p, f, r)}<K+\varepsilon
$$

for all $r(0<r \leqq r(p))$. Then we may assume that $f(F)$ is contained in a parametric ball $B^{\prime}=\left(B^{\prime}, \phi\right)$ in $N$ such that $\phi(f(p))=0$. Then it follows from (c) that

$$
C_{N}\left(A^{*}(p, r)\right) \geqq \tau_{N}{ }^{-n} C\left[\phi\left(A^{*}(p, r)\right)\right] .
$$

On the other hand there exist $q_{1}{ }^{\prime}$ and $q_{2}{ }^{\prime}$ in $B^{\prime}$ such that

$$
d_{N}\left(f(p), q_{1}{ }^{\prime}\right)=l(p, f, r) \text { and } d_{N}\left(f(p), q_{2}{ }^{\prime}\right)=L(p, f, r) .
$$

Since

$$
\left|\phi\left(q_{2}{ }^{\prime}\right)\right| /\left|\phi\left(q_{1}{ }^{\prime}\right)\right| \leqq \tau_{N} \frac{L(p, f, r)}{l(p, f, r)}<\tau_{N}(K+\varepsilon),
$$


it follows from Theorem 1 that

$$
C\left[\psi\left(A^{*}(p, r)\right)\right] \geqq \mathscr{H}_{n}\left(\left|\psi\left(q_{2}{ }^{\prime}\right)\right| /\left|\psi\left(q_{1}{ }^{\prime}\right)\right|\right) \geqq \mathscr{H}_{n}\left(\tau_{N}(K+\varepsilon)\right)>0 .
$$

Hence we can choose $\tau_{N}{ }^{-n} \mathscr{H}_{n}\left(\tau_{N}(K+\varepsilon)\right)$ as $\alpha$.

Conversely suppose there exists a finite constant $\alpha>0$ with the property in the theorem. First we assume that $H(p, f)>\tau_{N}$. Then there exists a decreasing sequence of real numbers $\left\{r_{j}\right\}_{j=1}^{\infty}$ such that $r_{j} \rightarrow 0$ as $j \rightarrow \infty, L\left(p, f, r_{j}\right) / l\left(p, f, r_{j}\right)$ $\rightarrow H(p, f)$ as $j \rightarrow \infty$ and $L\left(p, f, r_{\jmath}\right) / l\left(p, f, r_{\jmath}\right)>\tau_{N}$ for all $\jmath$. Then it follows from Lemma 3 that

$$
0<\alpha \leqq C_{N}\left(A^{*}\left(p, r_{\jmath}\right)\right) \leqq \tau_{N}{ }^{n} \frac{\omega_{n-1}}{\left(\log \tau_{N}{ }^{-1} \frac{L\left(p, f, r_{\jmath}\right)}{l\left(p, f, r_{\jmath}\right)}\right)^{n-1}} .
$$

This implies that

$$
\frac{L\left(p, f, r_{j}\right)}{l\left(p, f, r_{j}\right)} \leqq \tau_{N} \exp \left\{\left(\frac{\tau_{N}^{n} \cdot \omega_{n-1}}{\alpha}\right)^{1 /(n-1)}\right\} .
$$

By letting $\jmath \rightarrow \infty$, we have

$$
H(p, f) \leqq \tau_{N} \exp \left\{\left(\frac{\tau_{N}^{n} \cdot \omega_{n-1}}{\alpha}\right)^{1 /(n-1)}\right\} .
$$

Hence we always have the same inequality. This completes the proof.

Remark. Theorem 3 is a generalization of Theorem 1 in $[1]$ to the case of Riemannian manifolds.

\section{Main result}

For a non-compact Riemannian manifold $M$ we denote by $R(M)$ the class of all bounded $A C L$ funcitons $f$ on $M$ which have finite Dirichlet integral $D_{M}(f)$. $<\infty$. Then $R(M)$ constitutes an algebra over the field of real numbers in a usual way and is called the Royden algebra associated with $M$. The Royden compactification of $M$ is denoted by $M^{*}$ (cf. [5], [6], [8], [10]).

Let $M$ and $N$ be two Riemannian manifolds of dimension $n(n \geqq 2)$. Let $f: M \rightarrow N$ be a homeomorphism. Then we have the following lemma.

LEMMA 5. The following conditions are equivalent.

(i) $f$ can be extended to a homeomorphism of $M^{*}$ onto $N^{*}$.

(ii) Let $X$ and $Y$ be any subsets of $M$. Then $\bar{X} \cap \bar{Y}=0$ in $M^{*}$ if and only if $\overline{f(X)} \cap \overline{f(Y)}=0$ in $N^{*}$.

(iii) Let $A$ be any generalized ring in $M$. Then $C_{M}(A)<\infty$ if and only if $C_{M}(f(A))<\infty$.

Remark. Any homeomorphism $f: M^{*} \rightarrow N^{*}$ induces a homeomorphism $f \mid M$ : $M \rightarrow N$ satisfying (iii) which is called a Royden's map in $[9,11]$. 
THEOREM 4. Let $M$ and $N$ be two Rremannian manifolds of dimension $n$ $(\geqq 2)$. If there exists a homeomorphism $f$ of $M^{*}$ onto $N^{*}$, then there exists a compact subset $K$ of $M$ such that the restruction of $f$ to each component of $M-K$ is quasiconformal.

Proof. Suppose the theorem were not the case. Then we could find a compact exhaustion $\left\{K_{j}\right\}_{j=1}^{\infty}$ of $M$ such that $\sup _{p \in M-K_{j}} H(p, f)=\infty$ for every $\jmath$. Hence there exsits a sequence $\left\{p_{j}\right\}_{j=1}^{\infty}$ of points in $M$ such that

$$
H\left(p_{\jmath}, f\right)>\tau_{N} \exp \left(j^{2 /(n-1)}\right) \quad\left(>\tau_{N}\right) .
$$

We may assume that $\left\{p_{j}\right\}_{j=1}^{\infty}$ is a discrete set.

For each $j$, there is a sequence $\left\{r_{\nu}\right\}_{\nu=1}$ of real numbers such that $r_{\nu} \rightarrow 0$ as $\nu \rightarrow \infty$ and

$$
\frac{L\left(p_{\jmath}, f, r_{\nu}\right)}{l\left(p_{\jmath}, f, r_{\nu}\right)}>\tau_{N} \exp \left(j^{2 /(n-1)}\right) \quad\left(>\tau_{N}\right)
$$

for all $\nu=1,2, \cdots$. Then we may assume that $\left\{q^{\prime} \in N: d_{N}\left(f\left(p_{\jmath}\right), q^{\prime}\right) \leqq r_{\nu}\right\}$ is contained in a parametric ball $B_{\jmath}^{\prime}=\left(B_{\jmath}^{\prime}, \phi_{\jmath}{ }^{\prime}\right)$ for sufficiently large $\nu$. Then it follows from the Proposition that

$$
C_{M}\left[f^{-1}\left(A^{*}\left(p_{\jmath}, f, r_{\nu}\right)\right)\right] \geqq \tau_{M}^{-n} \mathcal{H}_{n}\left(\tau_{M}^{2}\right)>0
$$

for sufficiently large $\nu$. We may assume that $\phi_{j}\left(f\left(p_{\jmath}\right)\right)=0$. Since $L\left(p_{\jmath}, f, r_{\nu}\right) /$ $l\left(p_{\jmath}, f, r_{\nu}\right)>\tau_{N}$ for sufficiently large $\nu$, we obtain that

$$
C_{N}\left(A^{*}\left(p_{\jmath}, f, r_{\nu}\right)\right) \leqq \tau_{N}{ }^{n} C\left[\phi_{\jmath}\left(A^{*}\left(p_{\jmath}, f, r_{\nu}\right)\right)\right]<\frac{\tau_{N}^{n} \cdot \omega_{n-1}}{j^{2}} .
$$

This shows that, for each $j$, there exists a sufficiently small $r$, such that $\left\{A^{*}\left(p_{\jmath}, f, r_{\jmath}\right)\right\}_{j=1}^{\infty}$ is a distinguished family in $N$ and

$$
\begin{aligned}
& C_{M}\left[f^{-1}\left(A^{*}\left(p_{\jmath}, f, r_{\jmath}\right)\right)\right] \geqq \tau_{M}{ }^{-n} \cdot \mathscr{H}_{n}\left(\tau_{M}^{2}\right)>0, \\
& C_{N}\left(A^{*}\left(p_{\jmath}, f, r_{\jmath}\right)\right)<\frac{\tau_{M}^{n} \cdot \omega_{n-1}}{j^{2}} .
\end{aligned}
$$

We set

and

$$
C_{0, \jmath}=\left\{q^{\prime} \in N ; d_{N}\left(f\left(p_{\jmath}\right), q^{\prime}\right) \geqq L\left(p_{\jmath}, f, r_{\jmath}\right)\right\}
$$

$$
C_{1, \jmath}=\left\{q^{\prime} \in N ; d_{N}\left(f\left(p_{\jmath}\right), q^{\prime}\right) \leqq l\left(p_{\jmath}, f, r_{\jmath}\right)\right\} .
$$

Furthermore if we set

$$
C_{0}=\bigcap_{\jmath=1}^{\infty} C_{0, \jmath} \text { and } C_{1}=\bigcup_{\jmath=1}^{\infty} C_{1 \jmath},
$$

then $A=R\left(C_{0}, C_{1} ; N\right)$ is a generalized ring. Since $f^{-1}(A)=R\left(f^{-1}\left(C_{0}\right), f^{-1}\left(C_{1}\right) ; M\right)$ is also a generalized ring, it follows from Lemma 4 that 


$$
C_{N}(A)=\sum_{\jmath=1}^{\infty} C_{N}\left(A^{*}\left(p_{\jmath}, f, r_{\jmath}\right)\right)
$$

and

$$
C_{M}\left(f^{-1}(A)\right)=\sum_{\jmath=1}^{\infty} C_{M}\left[f^{-1}\left(A^{*}\left(p_{\jmath}, f, r_{\jmath}\right)\right)\right]=\infty .
$$

This contradicts (iii) in Lemma 5. Hence there exists a compact set $K$ in $M$ such that $f$ is quasiconformal on each component of $M-K$.

For Riemann surfaces we can prove the following sharp theorem. However we do not know whether this is valid for higher dimensional cases.

THEOREM 5. Let $M$ and $N$ be two Riemann surfaces. If there exists a homeomorphism of $M^{*}$ onto $N^{*}$, then there exists a quasiconformal mapping of $M$ onto $N$.

Proof. Let $f$ be a homeomorphism of $M^{*}$ onto $N^{*}$. By Theorem 4, there exists a compact set $K$ in $M$ such that the restriction of $f$ to each component of $M-K$ is a quasiconformal mapping. Then we can find a compact bordered surface $R$ of $M$ such that $K \subset \bar{R} \subset M$. If we set $S=f(R)$, then the borders $\partial R$ and $\partial S$ consist of a finite number of disjoint quasiconformal curves (cf. [4, p. 101]). By a slight modication of the proof of Satz 8.2 in [4] we can find a quasiconformal mapping $f_{1}$ of $R$ onto $S$ such that $f=f_{1}$ in a neighborhood of $\partial R$. By setting $g=f_{1}$ in $R$ and $=f$ on $M-K$, we have a desired quasiconformal mapping.

\section{REFERENCES}

[1] P. CARAman, Characterization of quasiconformality by arc families of extremal length zero. Ann. Acad. Sci. Fenn. Ser. A I, 528, (1973), 1-10.

[2] F. Gehring, Symmetrization of rings in space. Trans. Amer. Math. Soc., 101 (1961), 499-519.

[3] F. Gehring, Rings and quasiconformal mappings in space. Trans. Amer. Math. Soc., 103 (1962), 353-393.

[4] O. Lehto ANd K.I. Virtanen, Quasikonforme Abbildungen. Springer 1965.

[5] J. LeLong-Ferrand, Etude d'une classe d'applications liées à des homomorphismes d'algebras de fonctions, et generalisant les quasi conformes. Duke Math. J., 40 (1973), 163-183.

[6] L.G. LEwis, Quasiconformal mappings and Royden algebras in space. Trans. Amer. Math. Soc., 158 (1971), 481-492.

[7] C. Loewner, On the conformal capacity in space. J. Math. Mech., 8 (1959), 411-414.

[8] M. NAKAI, Algebraic criterion on quasiconformal equivalence of Riemann surfaces. Nagoya Math. J., 16 (1960), 157-184.

[9] M. NakaI, Royden's map between Riemann surfaces. Bull. Amer. Math. Soc., 72 (1966), 1003-1005.

[10] M. NAKAI, Royden algebras and quasi-isometries of Riemannian manifolds. Pa- 
cific J. Math., 40 (1972), 397-414.

[11] L. SARIO AND M. NAKaI, Classification theory of Riemann surfaces. Springer 1970.

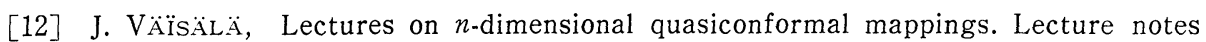
in mathematics, 229, Springer 1971.

Department of Mathematics Nagoya Institute of Technology Gokiso, Shôwa, Nagoya 466 JAPAN
Department of Mathematics

HOKKAIDO UNIVERSITY

SAPPORO, HoKKAIDO 060

JAPAN

Current Address

Department of Mathematics JoETSU UNIVERSITY

OF EduCATION

YAMAYASHIKI, Joetsu 943

JAPAN 